\title{
Neuere Untersuchungen zur Prädiktion von EEG-Signalen bei Epilepsie
}

\author{
C. Niederhöfer ${ }^{1}$ and R. Tetzlaff ${ }^{1}$ \\ ${ }^{1}$ Institut für Angewandte Physik, Johann Wolfgang Goethe Universität, Frankfurt, Deutschland
}

Zusammenfassung. Seit einigen Jahren ist die Analyse von EEG-Signalen bei Epilepsie Gegenstand zahlreicher wissenschaftlicher Arbeiten; Zielvorstellung ist dabei die Entwicklung von Verfahren zur Erkennung eines möglichen Voranfallszustandes. Im Vordergrund steht beispielsweise die Approximation einer so genannten effektiven Korrelationsdimension, die Bestimmung der maximalen LyapunovExponenten, Detektionsverfahren für Muster bei Zellularen Nichtlinearen Netzwerken, die Bestimmung der mittleren Phasenkohärenz und Verfahren zur nichtlinearen Prädiktion von EEG-Signalen. Trotz umfangreicher Bemühungen kann bis heute eine Erkennung von Anfallsvorboten mit einer Sensitivität und Spezifität, die eine automatisierte Anfallsvorhersage ermöglichen würde, noch nicht durchgeführt werden. In diesem Beitrag werden neue Ergebnisse zur Prädiktion von EEG-Signalen bei Epilepsie vorgestellt. Dabei werden Signale, welche mittels intrakranieller electrocorticographischer $(\mathrm{ECoG})$ und stereoelectroencephalographischer (SEEG) Ableitungen registriert wurden, segmentweise analysiert. Unter der Annahme, dass sich Änderungen des Systems „Gehirn” als Änderungen im Prädiktor, d.h. in seinen Systemparametern widerspiegeln, könnte eine nähere Betrachtung der Prädiktoreigenschaften zu einer Erkennung von Anfallsvorboten führen.

\section{Einleitung und Grundlagen}

Aktuell leidet etwa $1 \%$ der Weltbevölkerung unter einer in unregelmäßigen Abständen wiederkehrenden neurologischen Störung genannt Epilepsie. Bei etwa der Hälfte dieser Patienten handelt es sich um eine so genannte fokale Epilepsie. Eine erhebliche Verbesserung des Zustandes kann bei einem kleinen Teil dieser Patienten durch eine Resek-

Correspondence to: C. Niederhöfer

(niederhoefer@iap.uni-frankfurt.de) tion des betroffenen Gehirnbereichs erzielt werden. Dabei kann davon ausgegangen werden, daß der anfallsgenerierende Bereich im Gehirn abgegrenzt und vor allem auch lokalisiert werden kann. Patienten bei denen ein operativer Eingriff nicht möglich ist, müssen sich auf eine medikamentöse Therapie zur Kontrolle ihrer Anfälle verlassen. Dabei treten nicht selten Nebenwirkungen wie psychische Anomalitäten oder aber Medikamentenadaption - Wirkungsverlust des Medikaments durch langfristige Anwendung - auf. In beiden Fällen muss die Behandlung umgestellt werden, bzw. eine neue Therapie gewählt werden, was zusätzliche Belastungen für den Patienten bedeutet.

Zur Realisierung eines implantierbaren Systems zur Warnung vor epileptischen Anfällen müssen Anfallsvorboten in einer EEG-Signalanalyse gefunden werden, mit welchen eine verlässliche Aussage über bevorstehende Anfallsereignisse getroffen werden können. In vorangehenden Arbeiten wurden bereits verschiedene Verfahren zur Bestimmung von Anfallsvorboten vorgestellt. Darunter waren Ansätze mit Volterra-Systemen (Niederhöfer et al., 2002), sowie verschiedene auf Zellularen Nichtlinearen Netzwerken $(\mathrm{CNN})$ basierende Verfahren (Niederhöfer und Tetzlaff, 2005; Tetzlaff und Weiss, 2002; Gollas et al., 2004; Niederhöfer et al., 2003; Gollas und Tetzlaff, 2005).

Als Eingangsdaten für die hier betrachteten Netzwerke dienen segmentierte EEG Signale. Für jedes dieser Signalsegmente wird eine Signalprädiktion mit verschiedenen zeitdiskreten CNN (DTCNN) durchgeführt, was in direkter Konsequenz zu einer Sequenz von Prädiktoren, sowie von Prädiktorfehlern führt. Diese werden auf signifikante Änderungen vor einem epileptischen Anfall untersucht. In dieser Veröffentlichung werden Ergebnisse basierend auf einschichtigen, sowie mehrschichtigen, gedächtnisbehafteten DTCNN vorgestellt und diskutiert.

In Niederhöfer und Tetzlaff (2006) und Niederhöfer et al. (2006) wurden sogenannte Fehlerprofile vorgestellt. Diese stellen die räumliche Abhängigkeit des Fehlers zur La-

Published by Copernicus Publications on behalf of the URSI Landesausschuss in der Bundesrepublik Deutschland e.V. 


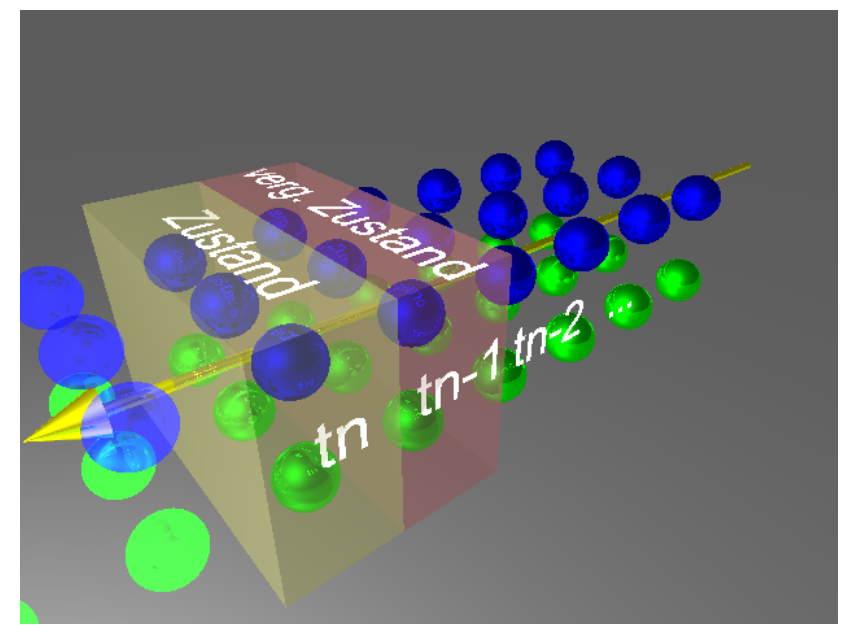

Abbildung 1. Schematische Darstellung eines zweischichtigen CNN mit $3 \times 1$ Zellen und $\tau=1$.

ge der Elektrode dar. Zu diesem Zweck wird mittels eines einfachen DTCNN mit linearen Gewichtsfunktionen für jeden Elektrodenpunkt (ein Sensor auf einem Elektrodenstreifen) der zeitliche Verlauf des Prädiktionsfehlers bestimmt und dann in einer zweidimensionalen Darstellung wiedergegeben. Hierbei werden die Elektrodenpunkte in der Reihenfolge aufgetragen wie sie auch im Datenformat vorkommen. Die Fehlerprofile ermöglichen es, eine oder mehrere signifikante Elektroden für tiefer gehende Untersuchungen herauszugreifen. Nach der Wahl einer geeigneten Elektrode oder, wenn möglich einem geeigneten Elektrodenpunkt, wird das EEG-Signal dieser Elektrode zur Prädiktion mit unterschiedlichen Netzwerken herangezogen. Zur Bewertung wird bei der Untersuchung der verschiedenen Prädiktoren der Prädiktionsfehler verwendet.

Die Zellzustandsgleichung

$x_{i}\left(t_{n+1}\right)=-x_{i}\left(t_{n}\right)+\sum_{\tau=0}^{T} \sum_{k=1}^{K} \sum_{j \in \mathcal{S}_{i}(r)} a_{j}^{(k)(\tau)} x_{j}^{k}\left(t_{n-\tau}\right)$

beschreibt die Zelldynamik des einschichtigen zeitdiskreten Netzwerks, wobei $\tau$ die Gedächtnistiefe, $\mathrm{k}$ die Polynomordnung der Gewichtsfunktionen und $\mathcal{S}_{i}(r)$ die Nachbarschaft der betrachteten Zelle festlegt. Zur Optimierung der Kopplungsgewichte wurde als Kriterium der Prädiktorfehler gemäß

$$
e_{1}(m)=\sqrt{\frac{1}{I * N} \sum_{n} \sum_{i} \frac{\left(x_{i}\left(t_{n}\right)-\hat{x}_{i}\left(t_{n}\right)\right)^{2}}{x_{i}^{2}\left(t_{n}\right)}}
$$

verwendet mit $\hat{x}_{i}$ als dem geschätzten Wert an der Zelle $i$ und $N$ als Anzahl der Werte pro Intervall. Für das ebenfalls im folgenden betrachtete Netzwerk mit 2 Schichten, führt eine einfache Modifikation von Gl. 1 zu

$x_{i}^{l}\left(t_{n+1}\right)=-x_{i}^{l}\left(t_{n}\right)+\sum_{\tau=0}^{T} \sum_{k=1}^{K} \sum_{j \in \mathcal{S}_{i}(r)} a_{j}^{(k)(\tau) l^{\dagger} l} x_{j}^{l^{\dagger} k}\left(t_{n-\tau}\right)$.

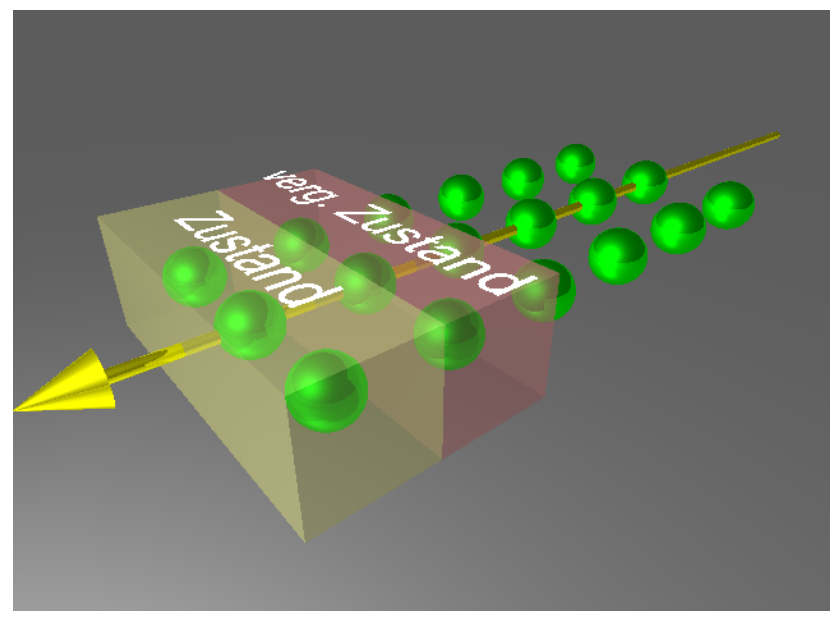

Abbildung 2. Schematische Darstellung eines einschichtigen CNN mit $3 \times 1$ Zellen und $\tau=1$.

$a_{j}^{(k)(\tau) l^{\dagger} l}$ definiert hierbei die Kopplung von der Schicht $l^{\dagger}$ in die Schicht $l$ und die Nachbarschaftsmenge $\mathcal{S}_{i}(r)$ bezieht beide Schichten mit ein (siehe Abb. 1). Hierbei wird als Optimierungskriterium ein erweiterter Prädiktorfehler gemäß

$e_{2}(m)=\sqrt{\frac{1}{N|\mathcal{R}|} \sum_{n=1}^{N} \sum_{\left(j, \ell^{\dagger}\right) \in \mathcal{R}} \frac{\left(x_{j}^{\ell^{\dagger}}\left(t_{n}\right)-\hat{x}_{j}^{\ell^{\dagger}}\left(t_{n}\right)\right)^{2}}{x_{j}^{\ell^{\dagger}}\left(t_{n}\right)}}$

benutzt.

Die zugrunde gelegte Zellausgangsfunktion ist in beiden hier betrachteten Fällen die sogenannte Identitätsfunktion $i d(x)$. Im folgenden werden 2 unterschiedliche Prädiktionsansätze untersucht.

- Ein einschichtiges CNN mit $3 \times 1$ Zellen und linearen Gewichtsfunktionen $(K=1)$, sowie einer Gedächtnistiefe $\tau=1$ (siehe Abb. 2)

- Ein zweischichtiges CNN mit 2 mal $3 \times 1$ Zellen und nichtlinearen Gewichtsfunktionen $(K=3)$, sowie einer Gedächtnistiefe $\tau=1$.

\section{Datenbasis}

In diesem Beitrag werden Ergebnisse präsentiert die auf EEG-Signalen basieren. Diese EEG-Signale wurden in der Klinik für Epileptologie in Bonn aufgezeichnet und dem Institut für Angewandte Physik zur Verfügung gestellt. Es handelt sich bei den Daten um kontinuierlich aufgezeichnete hirnelektrische Aktivität, welche im Rahmen von SEEG und ECoG Messungen registriert wurden. Dazu wurden verschiedenen Patienten Elektroden implantiert, welche insgesamt Ableitungen von bis zu 80 Elektrodenpunkten erzeugen.

Die Daten wurden mit einer Abtastrate von $200 \mathrm{~Hz}$ aufgezeichnet und in aufeinander folgende Segmente mit je 2000 Werten aufgeteilt, die jeweils einem Zeitintervall von 10 Sek. 

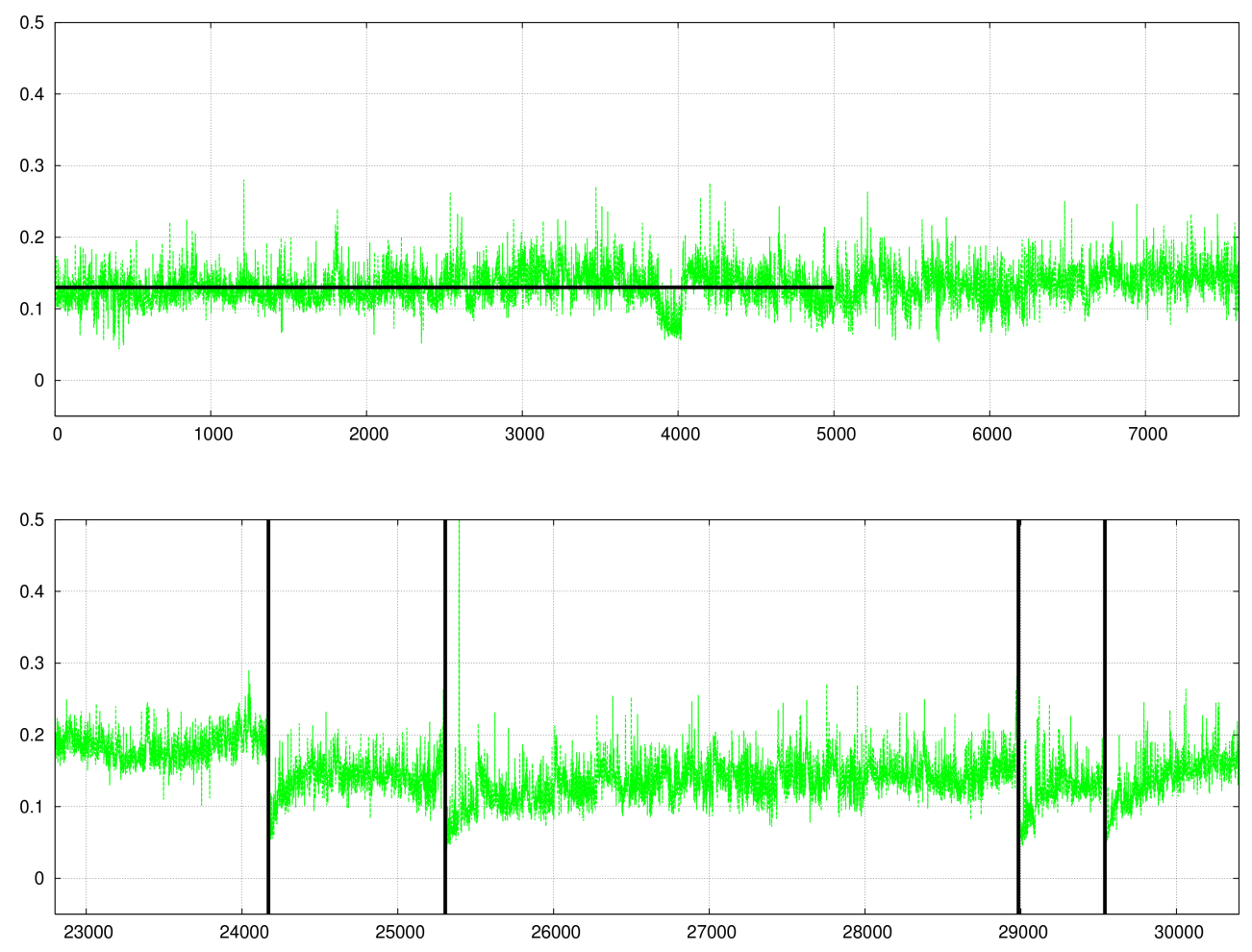

Abbildung 3. Oben: Verlauf von $e_{1}(m)$ in Abhängigkeit von Segmentnummer $m$ (anfallsfreier Bereich). Unten: Verlauf von $e_{1}(m)$ des Elektrodenpunkts TLL04 (Streifenelektrode) in Abhängigkeit von Segmentnummer $m .4$ Anfälle. Nach jedem Anfall ist ein Einbruch von $e_{1}(m)$ zu beobachten, danach ein stetiger Anstieg des Fehlers über den Ruhemittelwert bis zum nächsten Anfallsbeginn. Anfälle sind durch vertikale schwarze Linien markiert.

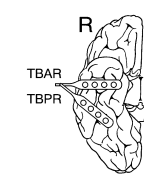

Streifenelektrode mit 4 Elektrodenpunkten

Streifenelektrode mit 6 Elektrodenpunkten

Tiefenelektrode mit 10 Elektrodenpunkten

Tabelle 1. Implantationsschema.

entsprechen. In einem einzigen Vorverarbeitungsschritt werden die Signale auf einen Wertebereich von $-1 \leq x \leq 1$ normiert. Zur möglichen Erkennung von Anfallsvorboten wurden ein- und zweischichtige Netzwerke analysiert.

\section{Einschichtige lineare Netzwerke}

Für das Netzwerk mit nur einer Schicht und den linearen Gewichtsfunktionen wurde aus dem vorher bestimmten Fehler- profil ein Elektrodenpunkt herausgegriffen und der Verlauf des Prädiktionsfehlers untersucht. In der interiktualen Phase, also dem Zeitraum in dem kein Anfall - unabhängig ob klinisch oder subklinisch - stattfindet, kann ein sogenannter Ruhemittelwert errechnet werden. Dazu werden aus der interiktualen Phase die ersten 5000 berechneten Prädiktionsfehler herangezogen. Es zeigt sich, daß der Prädiktionsfehler während der folgenden anfallsfreien Zeit nur sehr gering abweicht oder diesen sogar unterschreitet. Bei voranschreitender Zeit kann festgestellt werden, daß der Prädiktionsfehler vor einem Anfallsbeginn über den vorher bestimmten $R u$ hemittelwert $e_{1 m} \approx 0.13$ ansteigt, im Anfallsbeginn kurzzeitig einen größeren Fehler aufweist und direkt im Anschluss an den Anfallsbeginn ein erheblicher Abfall im Verlauf der Fehlers beobachtet werden kann. Nach diesem Abfall steigt der Prädiktionsfehler vor einem weiteren Anfallsbeginn wieder über den Ruhemittelwert an.

Dieses Verhalten ist in den beiden Teilabbildungen der Abb. 3 dargestellt. Der obere Teil zeigt normale also anfallsfreie Hirnaktivität an, der untere Teil dagegen stellt das zeitliche Verhalten des Fehlers $e_{1}(m)$ während eines Datensatzes mit 4 Anfällen dar. Der Ruhemittelwert wurde aus den ersten 5000 Werten des anfallsfreien dargestellten Zeitraums berechnet. Während des Zeitraums in dem die 4 Anfälle auftreten ist vor jedem Anfall der Anstieg von $e_{1}(m)$ über den 

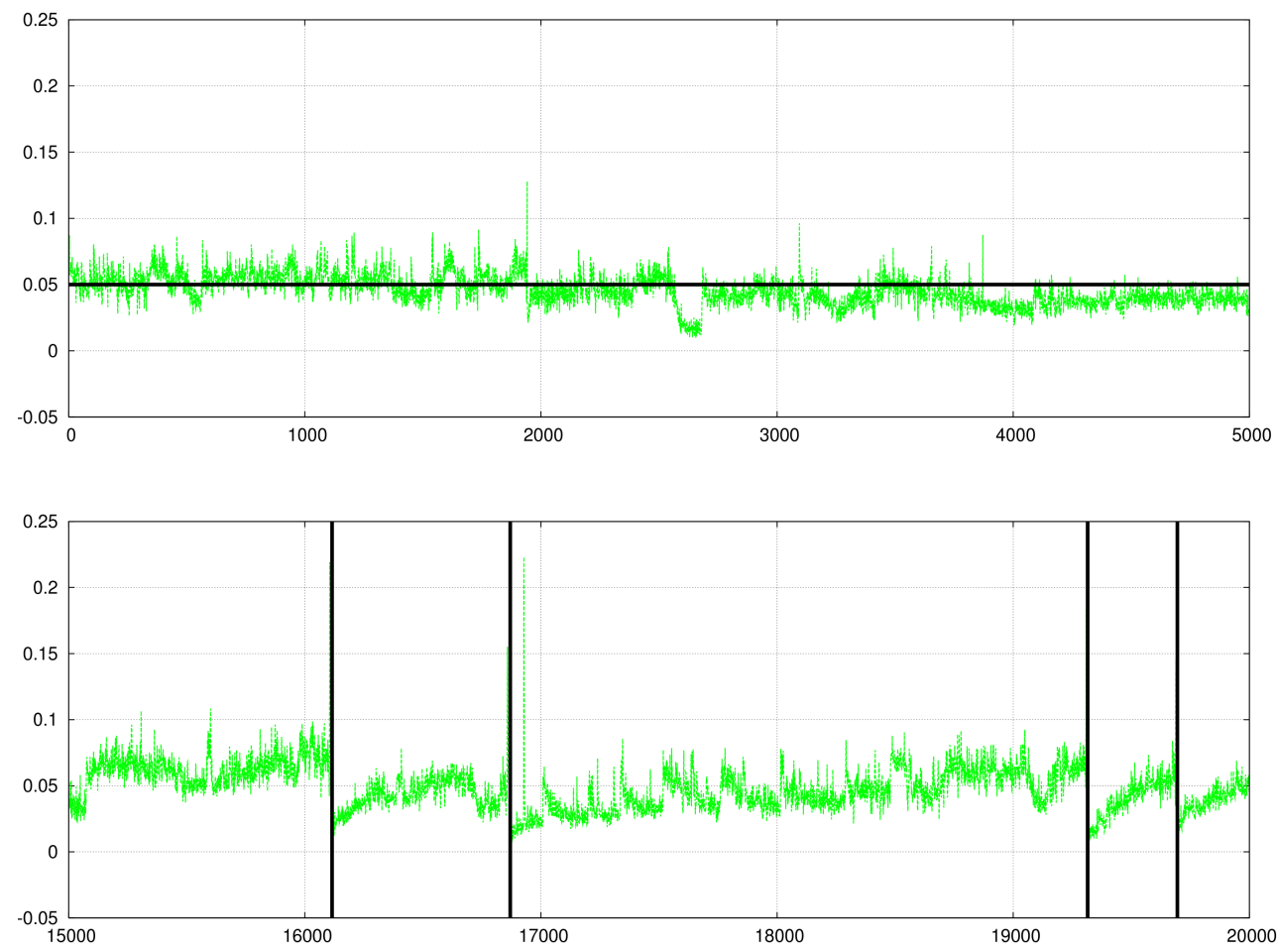

Abbildung 4. Oben: Verlauf von $e_{2}(m)$ in Abhängigkeit von Segmentnummer $m$ (anfallsfreier Bereich). Unten: Verlauf von $e_{2}(m)$ der Elektrodenpunktpaarung TLL02 (Streifenelektrode) und TL02 (Tiefenelektrode) in Abhängigkeit von Segmentnummer $m$. 4 Anfälle. Nach jedem Anfall ist ein Einbruch von $e_{2}(m)$ zu beobachten, danach ein stetiger Anstieg des Fehlers über den Ruhemittelwert bis zum nächsten Anfallsbeginn. Anfälle sind durch vertikale schwarze Linien markiert.

Ruhemittelwert zu erkennen.

\section{Zweischichtige nichtlineare Netzwerke}

Im zweiten untersuchten Fall wurde ein Netzwerk mit 2 Schichten und polynomialen Gewichtsfunktionen $(k=3)$ als Prädiktorsystem ausgewählt. Diese spezielle Netzwerkstruktur basiert auf der Annahme, daß sich während eines epileptischen Anfalls die Art der Kopplung zwischen einzelnen Hirnbereichen verändert, also demzufolge die Art der Kopplung zwischen den 2 Netzwerkschichten. In vorliegenden Fall wurde zur Bestimmung eines möglichen Voranfallszustands ebenfalls der Prädiktionsfehler $e_{2}(m)$ herangezogen, um einen Vergleich beider Prädiktorsysteme zu ermöglichen.

Die Vorverarbeitung der EEG-Signale ist identisch, abgesehen von der verwendeten Segmentlänge. Hier kommen Segmente von 15 Sek. Länge (3000 Werte) zur Anwendung. Der Fehler $e_{2}(m)$ wurde gemäß Gollas et al. (2004) definiert. Analog zur Messung mit dem einschichtigen Netzwerk wird auch hier ein Ruhemittelwert bestimmt. Dieser liegt bei ca. $e_{2 m} \approx 0.05$ und wurde ebenfalls aus den ersten 5000 Segmenten der anfallsfreien Phase bestimmt. Abbildung 4 zeigt im unteren Teil ein ähnliches Verhalten, wie es auch im Fall des einschichtigen Netzwerks beobachtet werden konn- te. Vor dem Beginn eines epileptischen Anfalls steigt der erreichte Prädiktionsfehler über den Ruhemittelwert hinweg an um direkt nach dem Anfallsbeginn erheblich abzufallen. Zum nächsten Anfall hin ist auch hier eine erneute Steigerung des Wertes von $e_{2}(m)$ über den vorher bestimmten $\mathrm{Ru}$ hemittelwert zu beobachten.

\section{Diskussion und Ausblick}

In dieser Veröffentlichung wurden 2 unterschiedlich komplexe Algorithmen aus dem Bereich der Prädiktion von EEGSignalen untersucht. Das zeitliche Verhalten der Prädiktionsfehler $e_{1}(m)$ und $e_{2}(m)$ in der anfallsfreien Phase und in der Voranfallsphase wurde untersucht und ein mögliches $\mathrm{Ma}$ zur Vorhersage von epileptischen Anfällen basierend auf den erhaltenen Prädiktionsfehlern definiert. Es wurde gezeigt, daß sowohl mit dem zweischichtigen Netzwerk als auch mit dem einfacheren einschichtigen Netzwerk recht gut übereinstimmende Ergebnisse erzielt werden konnten. Veränderungen an den Kopplungen zwischen den Schichten des zweischichtigen Netzwerks, die auf einen bevorstehenden Anfall hindeuten, konnten in den bisher betrachteten Fällen nicht beobachtet werden. Untersuchungen an weiteren Patientendaten werden zur Zeit durchgeführt. 
Acknowledgements. Das Projekt wurde von der DFG gefördert. Die Autoren danken Klaus Lehnertz von der Klinik für Epileptologie in Bonn für die zur Verfügung gestellten EEG-Signalaufzeichnungen.

\section{Literatur}

Niederhöfer, C., Suna, S., and Tetzlaff, R.: Nonlinear Prediction of Brain Electrical Activity in Epilepsy with a Volterra RLS Algorithm, Proceedings of the International Symposium on Circuits and Systems, Phoenix, Arizona, USA, 2002.

Niederhöfer, C. and Tetzlaff, R.: Prediction of Epileptic Seizures using Multi-Layer Delay-Type Discrete Time Cellular Nonlinear Networks (DTCNN) - Long-Term Studies, Proceedings of SPIE's Microtechnologies for a New Millenium, Sevilla, Spain, 2005.

Tetzlaff, R. and Weiss, D.: Cellular Neural Networks for the Anticipation of Epileptic Seizures, Proceedings of the International Symposium on Circuits and Systems, 2002.

Gollas, F., Niederhöfer, C., and Tetzlaff, R.: Prediction of Brain Electrical Activity in Epilepsy using a Higher Dimensional Prediction Algorithm for Discrete Time Cellular Neural Networks (DTCNN), Circuits and Systems, 2004. ISCAS '04. Proceedings of the 2004 International Symposium, Vancouver, 5, Canada, V720-V-723, 2004.
Niederhöfer, C., Fischer, P., and Tetzlaff, R.: Feature Extraction in Epilepsy using a Cellular Neural Network based Device - First Results, Proceedings of the International Symposium on Circuits and Systems, Bankok, Thailand, 2003.

Gollas, F. and Tetzlaff, R.: Modeling Complex Systems by Reaction-Diffusion Cellular Nonlinear Networks with Polynomial Weight Functions, Cellular Neural Networks and Their Applications CNNA, 2005 9th International Workshop, Hsin-Chu, Taiwan, 2005.

Niederhöfer, C. and Tetzlaff, R.: Detection of a preseizure state in epilepsy: Signal prediction by maximally weakly nonlinear networks?, Proceedings of the International Symposium on Circuits and Systems, Kos, Greece, 2006.

Niederhöfer, C., Gollas, G., and Tetzlaff, R.: Prediction Error Profiles allowing a seizure forecasting in epilepsy?, Cellular Neural Networks and Their Applications CNNA, 2006 10th International Workshop, Istanbul, Turkey, 2006. 